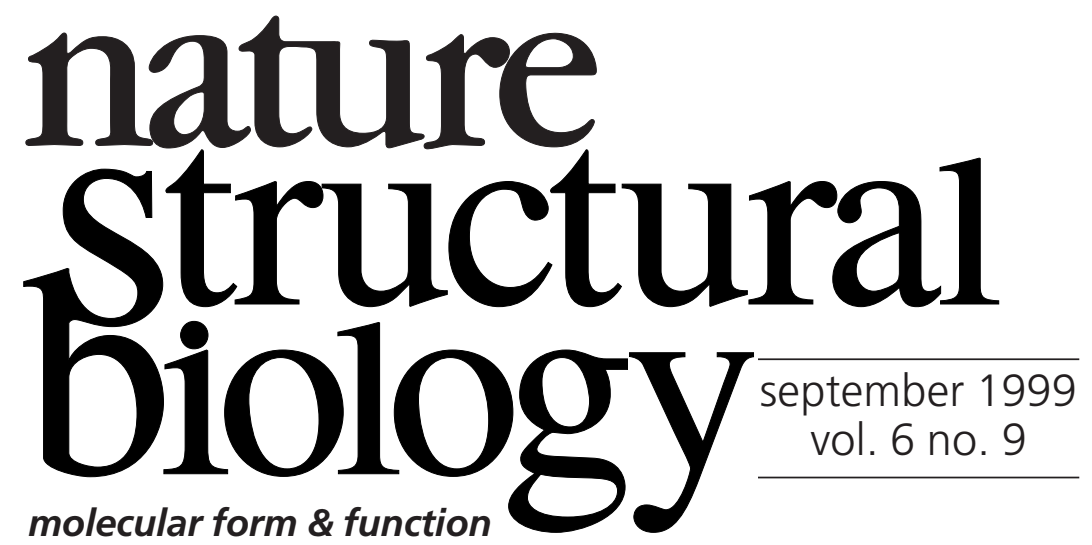

\title{
Synchrotrons in the news
}

Last year, Nature Structural Biology published a special supplement ${ }^{1}$ to accompany the August issue, highlighting the wide variety of biological research that benefits from the use of synchrotron radiation and the unprecedented worldwide increase in demand for use of synchrotron facilities. Biologists, especially crystallographers, have continued to clamor for beam time, and this demand will only increase in the future, thanks to the recently announced structural genomics initiatives in the USA, Japan and Germany ${ }^{2}$. Thus, it is heartening to see that in the year since the synchrotron supplement was published, proposals for increasing access to synchrotron facilities have advanced - and rapidly over the last few months. These plans have developed from interdepartmental and international cooperation to build, improve, or relocate facilities. Nevertheless, despite these positive moves, it is worth noting that the demand for beam time will likely continue to exceed capacity for some time to come.

\section{France and the United Kingdom cooperate}

Scientists in Europe are eager to have greater local access to cutting edge equipment and facilities to remain competitive. In the early 1990s, several proposals emerged for new third generation synchrotrons to be located in Europe, including the DIAMOND project in the UK and the SOLEIL project in France. However, on August 2, 1999 the French government announced ${ }^{3}$ that it will contribute financially to the construction and maintenance of DIAMOND, a medium energy synchrotron, with greater capabilities than the existing Synchrotron Radiation Source (SRS) at Daresbury, UK and the Laboratoire de l'Utilization du Rayonnement Électromagnétique (LURE) in Orsay, France. This collaboration will improve the scientific competitiveness of European scientists but leaves open the question of support for SOLEIL.

The expensive DIAMOND project was on hold for lack of funding until 1998, when the Wellcome Trust announced that it would contribute 110 million pounds sterling $(\sim 170$ million US dollars). This support was followed by a commitment from the Office of Science and Technology in the UK, which approved the project with an initial allocation of 35 million pounds sterling ( $\sim 57$ million US dollars) for the next few years. Now, France will contribute 350 million francs ( $\sim 57$ million US dollars) for the next seven years, plus an annual sum of 60-80 million francs ( $\sim 10-13$ million US dollars) for operation once the facility is complete. French scientists and engineers will participate in designing and building the facility, but the location of DIAMOND has not been decided, although the main proposal has been for the facility to be located in Daresbury, the present site of the SRS (Fig. 1). France emphasizes that selection of the site will remain the responsibility of Britain and notes that France will establish a section of the facility especially for French researchers.

The support of the French government for DIAMOND in the UK makes it unlikely that France will also support SOLEIL on its own soil. One reason behind the move to support a synchrotron elsewhere, according to the French government's press release ${ }^{3}$, is that France is eager to enhance scientific cooperation among European countries. Practically, it is unlikely that the French government will finance another expensive synchrotron. Thus, synchrotron users have reason to be both encouraged and disappointed. Financial support from three different groups ensures that DIAMOND will indeed be built (a fact that was in question two years ago). However, European biologists' demand for beam time is not likely to be satisfied without commitments to build additional synchrotrons in the near future. 


\section{editorial}

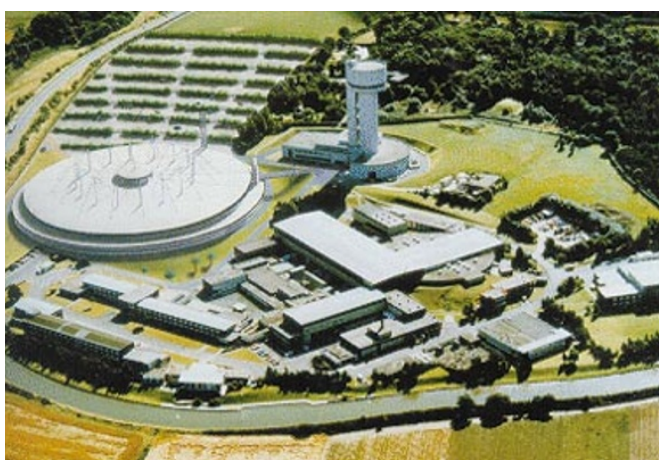

Fig. 1 Artist's impression of the DIAMOND facility (large ring at left) located on the SRS site in Daresbury, UK.
USA facilities get a booster shot of funds

The USA has a number of synchrotrons, but several must be refurbished to provide adequate technology and beam lines to meet the growing needs of the research community. The synchrotrons have traditionally been supported only by the Department of Energy (DOE) - but that has now changed. On July 21, 1999, the National Institutes of Health (NIH) announced ${ }^{4}$ a collaboration with the DOE to upgrade the Stanford Synchrotron Radiation Laboratory (SSRL) at Stanford University in California and the National Synchrotron Light Source (NSLS) at Brookhaven National Laboratory in New York.

The reason for NIH involvement is clear. Synchrotrons are oversubscribed, and recently biologists have been unable to obtain easy, prompt access to beam lines, often waiting six months or more to collect data. The commitment of 18 million US dollars (14 million to SSRL and 4 million to NSLS) from the NIH to improve these two synchrotrons will allow more efficient usage and will be a boost to $\mathrm{NIH}$-funded research, especially X-ray crystallography projects. Nevertheless, improvements at these synchrotrons will benefit not only biologists, but also scientists from other disciplines. This interdepartmental cooperation within the US government to support key research centers is sensible — and we would argue essential — to match the evolving research climate.

\section{Germany donates a synchrotron}

Germany has agreed to donate the BESSY-1 synchrotron, which is set to be replaced by a larger synchrotron, to scientists in the Middle East, a region that does not have a facility of its own. Germany is willing to absorb the cost of dismantling and moving the synchrotron, provided that a suitable location can be found and financial support for upgrading and maintaining the facility can be guaranteed. Five countries - Cyprus, Egypt, Iran, Palestine, and Turkey - have bid to host the synchrotron, and a decision on the location, which may be a difficult one politically, is possible in the next few months. However, it is likely that considerable financial support, from wealthier countries outside the Middle East, will be needed to allow the project to succeed. Both the European Union and the USA probably will be asked to underwrite the move (with US aid possibly coming from a portion of the Middle East aid package). However, they may need convincing to spend such a large sum on a research facility, given the great variety of basic human needs that exist in the region. For this reason, it should be noted that a vote of confidence for the Middle Eastern scientific community, in the form of this donated synchrotron, should engender more scientific cooperation and communication between countries - and would hopefully be a small step toward peace in the region. Notably, in an effort to meet the goal of widespread access and peaceful cooperation, Israel has abstained from bidding to host the synchrotron, since if it were housed in Israel it would most likely be off-limits to scientists in many other countries for political reasons.

\section{Fourth generation light sources}

Progress toward new synchrotron technology was seen in the announcement on April 6, 1999 of financial support from the DOE in the USA for research and design of a fourth generation light source, an X-ray free electron laser known as the Linac Coherent Light Source (LCLS), to be located at the Stanford Linear Accelerator Center (SLAC) site in California. Such a source could theoretically produce peak radiation 10 orders of magnitude brighter than that generated by current third generation synchrotrons $s^{5}$. However, much ground work must be accomplished to show that theory can be put to practice - work that is the responsibility of an LCLS research collaboration, consisting of scientists from the Stanford Linear Accelerator Center, Argonne National Laboratory, Brookhaven National Laboratory, University of California, Los Angeles, Lawrence Livermore National Laboratory, and Los Alamos National Laboratory. Research and design for the LCLS will be funded by the DOE for the next four years at a rate of 1.5 million US dollars annually. The knowledge gained from the X-ray free electron laser research is expected to benefit design and maintenance of existing synchrotron sources as well as help to solidify the conceptual design report for building the LCLS (which is due May, 2001). If all goes well, the 100 million US dollars required to build the LCLS and a few research stations could be requested as soon as 2001 .

\section{Rapid education}

Ten years ago, few biologists had heard of synchrotrons. Now they have become key resources for many biological researchers, and many more scientists have become educated about the need to support these multidisciplinary facilities. As seen from the sampling above, synchrotrons are in the scientific news almost weekly and should be the subject of much discussion for some time to come - as long as the need for access continues to outpace availability. 